\title{
DIFFERENTIAL CROSS SECTIONS FOR ELASTIC ELECTRON SCATTERING BY A CALCIUM ATOM AT LOW ENERGY RANGE.
}

\author{
Kinyanjui P.N., Ndeke W.M., Okumu J. and Singh C.S. \\ Department of Physics, Kenyatta University, \\ P.O. Box 43844-00100, Nairobi, Kenya \\ E-mail:william.ndeke@gmail.com/kinyanjuipaulngigi@gmail.com
}

\begin{abstract}
In this study the distorted wave method was applied in calculation of the differential cross sections (DCS) for elastic scattering of electron by a calcium atom at electron impact energies of 10, 15, 20, and $40 \mathrm{eV}$. At lower incident energies, 10, 15, and $20 \mathrm{eV}$ the present DCS results are not in good agreement with other theoretical and experimental results. However, at $40 \mathrm{eV}$ the present DCS results are in good agreement with other theoretical and experimental results.
\end{abstract}

Key words: Cross-sections, distortion potential, distorted wave, static potential and spherical harmonics.

DOI: $10.7176 /$ APTA/83-09

Publication date: September $29^{\text {th }} 2020$

\section{INTRODUCTION}

The studies of atomic collision processes have drawn immense attention since the introductory days of quantum mechanics. This has been occasioned by the fact that information about planetary processes is still insufficient. There is also need for detailed knowledge of atomic collisions in nuclear physics. To contribute to this, studies of atomic collisions have been carried out using different targets and projectiles. We have done a study on the lower energy range of collisions of calcium atom as the target and electrons.

Elastic scattering of electron by a calcium atom has been studied both experimentally and theoretically, and several results are available regarding these processes. Milisavljevic et al. (2005) used a crossed electron-atom beam experimental technique to measure differential and integral cross sections for elastic scattering of electron by a calcium atom at electron-impact energies of 10,20,40,60 and $100 \mathrm{eV}$ and for a range of scattering angles $(\Theta)$ from $10^{\circ}$ up to $150^{\circ}$. Raj et al. (2007) used complex optical potential method to calculate DCS for elastic scattering of electrons by a calcium atom at electron impact energies 10-500 eV. Pandya et al. (2010) used a complex optical potential to calculate DCSs for elastic electron scattering by a calcium atom at electron impact energies 10, 60 and $100 \mathrm{eV}$. The two theoretical approaches results when compared with experimental results do not agree well. As a result, in this study differential and integral cross sections for elastic electron-calcium scattering was calculated using distorted wave method in the energy range from $10-40 \mathrm{eV}$.

\section{THEORY}

Distorted wave method

Total Hamiltonian, H, for electron-calcium elastic collision is given as (Singh, 2004; Madison and Bartschat, 1996)

$\mathrm{H}=\mathrm{H}_{\mathrm{a}}+\mathrm{T}+\mathrm{V}$

Where $\mathrm{H}_{\mathrm{a}}$ is the Hamiltonian for the isolated atom, $\mathrm{T}$ is the kinetic energy operator for the projectile electron and $\mathrm{V}$ is the interaction between the projectile electron and the atom. 
The T-matrix $\left(T_{i f}\right)$ for the electron-target collision in the two potential approach is given by Madison and Bartschat (1996),

$$
\begin{aligned}
T_{i f}= & (\mathrm{N}+1)\left\langle\chi_{f}^{-}(0) \psi_{f}(1,----, \mathrm{N})\left|\mathrm{V}-U_{f}\right| \mathrm{A} \Psi_{i}^{+}(0,----, \mathrm{N})\right\rangle \\
& +\left\langle\chi_{f}^{-}(0) \psi_{i}(1,-----, \mathrm{N})\left|U_{f}\right| \psi_{i}(1,----, \mathrm{N}) \beta_{i}\right\rangle
\end{aligned}
$$

where $\beta_{i}$ is the initial plane wave given as,

$\beta_{i}=\exp \left(\mathrm{ik} \cdot \mathrm{r}_{0}\right)$

and $\psi_{i}$ and $\psi_{f}$ are respectively, the initial and final wave functions of the calcium atom.

When the Born series expansion is done for $\Psi_{i}^{+}$, and considering the first term of it only,

$$
\begin{aligned}
T_{i f}=(\mathrm{N}+1)\left\langle\chi_{f}^{-}(0) \psi_{f}(1,2, \ldots, \mathrm{N})\left|\mathrm{V}-U_{f}\right| \mathrm{A} \psi_{i}^{+}(1,2, \ldots, \mathrm{N}) \chi_{\mathrm{i}}^{+}(0)\right\rangle \\
+\left\langle\chi_{f}^{-}(0) \psi_{f}(1,2, \ldots, \mathrm{N})\left|U_{f}\right| \psi_{i}(1,2, \ldots, \mathrm{N}) \beta_{i}(0)\right\rangle
\end{aligned}
$$

When the target is one electron atom or quasi one electron atom (when we consider only one electron of the atom in the collision process) and elastic collision is being considered, then the direct matrix $\left(\mathrm{T}^{\mathrm{d}}\right)$ and exchange transition matrix ( $\mathrm{T}^{\mathrm{ex}}$ ) corresponding to the above $T_{i f}$ can be separated as,

$$
\begin{aligned}
\mathrm{T}^{\mathrm{d}}=\left\langle\chi_{f}^{-}(0) \psi_{i}(1)|V| \psi_{i}(1) \chi_{i}^{+}(0)\right\rangle \\
+\left\langle\chi_{f}^{-}(0)\left|U_{i}(0)\right| \beta_{i}(0)-\chi_{i}^{+}(0)\right\rangle
\end{aligned}
$$

and

$$
\begin{aligned}
\mathrm{T}^{\mathrm{ex}} & =\left\langle\chi_{f}^{-}(0) \psi_{i}(1)|V| \psi_{i}(0) \chi_{i}^{+}(1)\right\rangle \\
& -\left\langle\chi_{f}^{-}(0) \mid U_{i}(0) \psi_{i}(0)\right\rangle\left\langle\psi_{i}(1) \chi_{i}^{+}(1)\right\rangle
\end{aligned}
$$

since $\psi_{i}=\psi_{f}$ (for elastic collision process) and $U_{i}=U_{f}$. Here $\chi_{i}^{+}$is the distorted wavefunction representing the projectile electron in the initial channel and is a solution to the wave equation,

$\left(\nabla_{0}^{2}-U_{i}+k_{i}^{2}\right) \chi_{i}^{+}=0$

where $U_{i}$ is an arbitrarily chosen potential for the distortion of the initial state projectile electron and $k_{i}$ is the initial wave vector of the projectile electron. In this study $U_{i}=U_{f}$ and it has been taken as the initial state static potential of calcium atom.

\section{Distorted waves}

The distorted waves $\chi_{i}^{+}$and $\chi_{f}^{-}$for the projectile in the initial and final states are expanded in terms of partial waves (Singh, 2005) as, 
$\left|\chi_{i}^{+}\right\rangle=\sqrt{\frac{2}{\pi}} \frac{1}{k_{i} r} \sum_{l_{i} m_{i}} i^{l_{i}} \chi_{l_{i}}\left(k_{i}, \mathrm{r}\right) Y_{l_{i} m_{i}}(\hat{r}) Y_{l_{i} m_{i}}^{*}\left(\widehat{k}_{l}\right)$

and

$\left|\chi_{f}^{-}\right\rangle=\sqrt{\frac{2}{\pi}} \frac{1}{k_{f} r} \sum_{l_{f} m_{f}} i^{l_{f}} \chi_{l_{f}}^{*}\left(k_{f}, \mathrm{r}\right) Y_{l_{f} m_{f}}(\hat{r}) Y_{l_{f} m_{f}}^{*}\left(\widehat{k_{f}}\right)$

where $Y_{l m}$ are spherical harmonics.

\section{Cross sections}

The radial distorted wave equations for initial and final states will be solved using Numerov method and the differential cross sections will be obtained using the relation,

$\frac{d \sigma}{d \Omega}=4 \pi^{4} \frac{k_{f}}{k_{i}}\left(\frac{1}{4}\left|\mathrm{~T}^{\mathrm{d}}+\mathrm{T}^{\mathrm{ex}}\right|^{2}+\frac{3}{4}\left|\mathrm{~T}^{\mathrm{d}}-\mathrm{T}^{\mathrm{ex}}\right|^{2}\right)$

Which when integrated yields the total cross section $(\sigma)$ as,

$\sigma=\int \frac{d \sigma}{d \Omega} \mathrm{d} \Omega$

$\sigma=2 \pi \int_{0}^{\pi} \frac{d \sigma}{d \Omega} \sin \Theta \mathrm{d} \Theta$

\section{Distortion potential}

Since elastic scattering was being considered, both the initial and final distortion potentials were taken as the static potential of a calcium atom in the initial state that is,

$U_{i}=U_{f}=\left\langle\psi_{i}|V| \psi_{i}\right\rangle$

where $U_{i}$ and $U_{f}$ are initial and final distortion potentials respectively.

\section{RESULTS AND DISCUSSIONS}

From figure 1., it can be seen that the present results for the differential cross sections at $10 \mathrm{eV}$ are not in qualitative agreement with the experimental results of Milisavljevic et al. (2005), the present results have two minima at $35^{\circ}$ and $115^{\circ}$ whereas the measured results of Milisavljevic et al. (2005) and calculated optical potential results of Khare et al. (1985), Pandya et al. (2010) and Raj and Kumar (2007) have three minima. The minimum at $35^{\circ}$ is very shallow and the one at $115^{\circ}$ is shifted to the left when compared with experimental results of Milisavljevic et al. (2005).

At $15 \mathrm{eV}$, there are no available measured or calculated results to compare with. Figure 2 shows the present results with exchange potential (DWM WE) and present results without the exchange potential (DWM WOE). From $0^{\circ}$ $120^{\circ}$ DWM WE results are higher than DWM WOE results. This shows that at lower incident energies exchange effect is significant since interaction between projectile and the target is for a longer time.

At $20 \mathrm{eV}$, figure 3 shows that the present results for the differential cross sections are not in agreement with the experimental results of Milisavljevic et al. (2005). The present results has one minimum at $100^{\circ}$ whereas measured results of Milisavljevic et al. and calculated optical potential results of Khare et al. (1985), Pandya et al. (2010) and Raj and Kumar (2007) have three minima. This could be attributed to the fact that first order distorted wave method does not give good results at lower energies. In figure 4 it can be seen that at $40 \mathrm{eV}$ the present results are in qualitative but not quantitative agreement with experimental results of Milisavljevic et al. (2005). The present result has two minima as the measured results of Milisavljevic et al. (2005) and calculated optical potential results 
of Khare et al. (1985) and Raj and Kumar (2007). The positions of the two minima in the present result are the same as in the other theoretical and experimental results but the magnitude (values) are different.

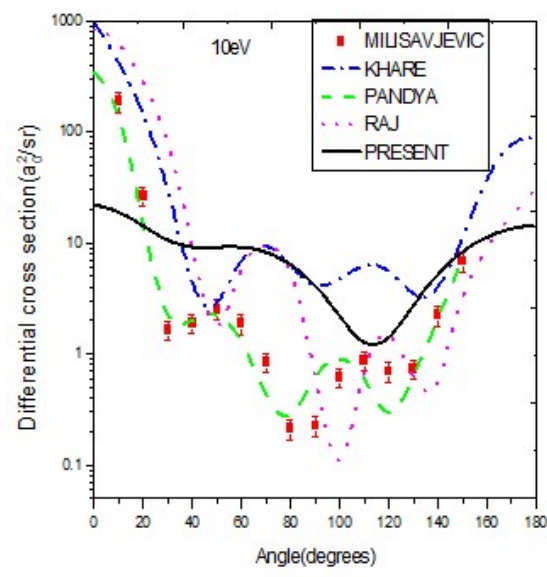

Figure 1: Differential cross sections for elastic scattering of electrons by a calcium atom at $10 \mathrm{eV}$ incident energy.

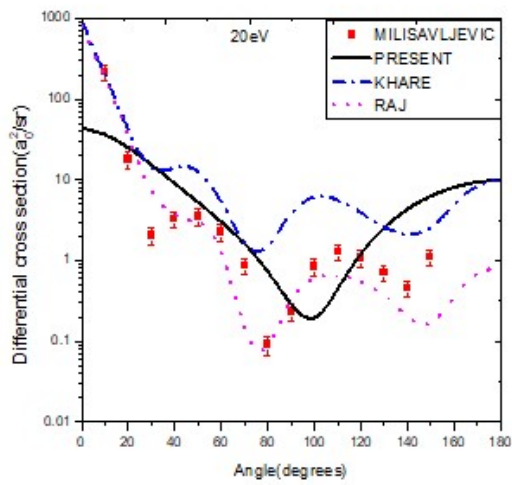

Figure 3: Differential cross sections

for elastic scattering of electrons by a calcium atom at $20 \mathrm{eV}$ incident energy.

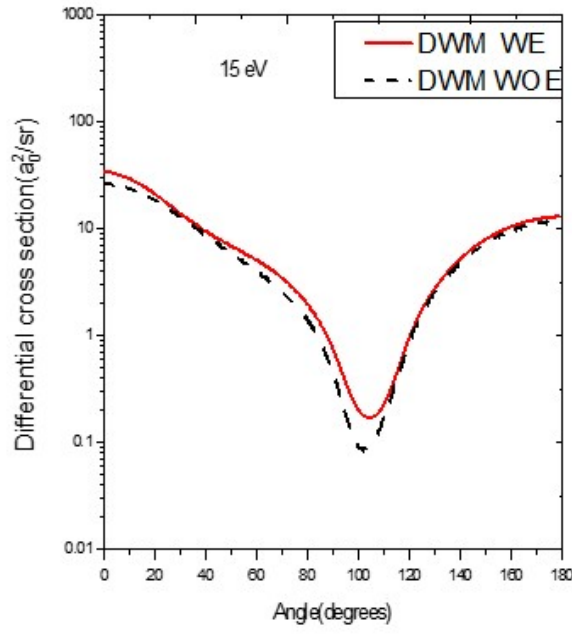

Figure 2: Differential cross sections for elastic scattering of electrons by a calcium atom at $15 \mathrm{eV}$ incident energy.

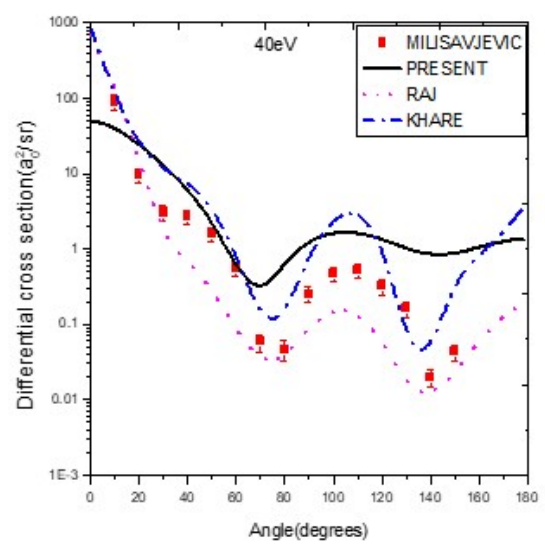

Figure 4: Differential cross sections for elastic scattering of electrons by a calcium atom at $40 \mathrm{eV}$ incident energy. 


\section{Conclusions}

At lower incident energies (10-20 eV), energies just above ionization threshold, the present DCS results are not in good agreement with measured results. This can be attributed to the fact that first order distorted wave method does not give good results at lower incident energies. At $40 \mathrm{eV}$, the present DCS results are in the better agreement with experimental results of Milisavljevic et al. (2005) compared to the calculated optical potential results of Khare et al. (1985), Raj and Kumar (2007) and Pandya et al. (2010).

\section{ACKNOWLEDGEMENT}

We want to thank the physics department, Kenyatta University for providing the needed facility to undertake this project.

\section{REFERENCES}

1. Bartschart, K. (1996). In Computational Atomic Physics; Electron and Positron Collisions with Atoms and Ions, Ed. Bartchart, K. (Springer- Verlag, Berlin) pp 10-12.

2. Khare, S.P., Ashok, K. and Vijayshri (1985). Elastic Scattering of Electrons and Positrons by the Calcium Atom. Journal of Physics B: Atomic, Molecular and Optical Physics 18: 1827-1840.

3. Madison, D.H. and Bartschart, K. (1996). The Distorted Wave Method for Elastic Scattering and Atomic Excitation, In Computation Atomic Physics. Ed. K. Bartschart. (Springer-Verlay, Berlin).

4. Milisavljevic, S., Sevic, D., Chauhan, R.K., Pejcev, V., Filipovic, D.M., Srivastava, R. and Marinkovic, B.P. (2005). Differential and Integrated Cross Sections for the Elastic Electron Scattering by Calcium Atom. Journal of Physics B: Atomic, Molecular and Optical Physics 38: 2371-2384.

5. Pandya, C.V., Patel, P.M. and Baluja, K.L. (2010). Differential Scattering Cross Sections for Elastic Electron Scattering by a Calcium Atom. Chinese Journal of Physics 48: 451-459.

6. Raj, D. and Kumar, A. (2007). Cross Sections for Electron Scattering by a Calcium Atom at Intermediate Energy. Journal of Physics B: Atomic, Molecular and Optical Physics 40: 3101-3111.

7. Singh, C.S. (2004). Magnetic Sublevel Differential Cross Sections for Electron Impact Excitation of $2{ }^{1} \mathrm{P}$ State of Helium. East African Journal of Physical Sciences 5: 85-98. 